\title{
Uncertainties of reverberation time estimation via adaptively identified room impulse responses
}

\author{
Lifu Wua) \\ Collaborative Innovation Center of Atmospheric Environment and Equipment Technology, \\ Nanjing University of Information Science and Technology, Nanjing, 210044, China \\ Xiaojun Qiu \\ School of Electrical and Computer Engineering, RMIT University, Melbourne, Australia \\ Ian Burnett \\ Faculty of Engineering and IT, University of Technology Sydney, Sydney, Australia \\ Yecai Guo \\ Collaborative Innovation Center of Atmospheric Environment and Equipment Technology, \\ Nanjing University of Information Science and Technology, Nanjing, 210044, China
}

(Received 24 August 2015; revised 7 February 2016; accepted 12 February 2016; published online 10 March 2016)

\begin{abstract}
This paper investigates the reverberation time estimation methods which employ backward integration of adaptively identified room impulse responses (RIRs). Two kinds of conditions are considered; the first is the "ideal condition" where the anechoic and reverberant signals are both known a priori so that the RIRs can be identified using system identification methods. The second is that only the reverberant speech signal is available, and blind identification of the RIRs via dereverberation is employed for reverberation time estimation. Results show that under the "ideal condition," the average relative errors in 7 octave bands are less than $2 \%$ for white noise and $15 \%$ for speech, respectively, when both the anechoic and reverberant signals are available. In contrast, under the second condition, the average relative errors of the blindly identified RIR-based reverberation time estimation are around $20 \%-30 \%$ except the $63 \mathrm{~Hz}$ octave band. The fluctuation of reverberation times estimated under the second condition is more severe than that under the ideal condition and the relative error for low frequency octave bands is larger than that for high octave bands under both conditions. (C) 2016 Acoustical Society of America. [http://dx.doi.org/10.1121/1.4943547]
\end{abstract}

$[\mathrm{MRB}]$

Pages: 1093-1100

\section{INTRODUCTION}

Reverberation time $\left(T_{60}\right)$ is defined as the time in seconds that is required for a steady-state sound level to decay $60 \mathrm{~dB}$ below its initial level after the sound source is abruptly turned off. ${ }^{1}$ It is an important parameter not only for characterizing the acoustic properties of a room, but also for designing a proper dereverberation method in many acoustic signal processing applications, so significant attention has been paid to $T_{60}$ estimation over the past several decades. ${ }^{2-9}$ Among these methods, the interrupted noise method $^{3}$ and the Schroeder's impulse response backward integration method ${ }^{4}$ (Schroeder's method) are two standard approaches. One problem of these two methods is that the excitation signals used may disturb or irritate nearby occupants, and the test rooms need to be unoccupied according to the ISO 3382 standard. ${ }^{3}$

To accomplish reverberation time measurement without disturbing audiences, a number of methods attempt to estimate reverberation time from the reverberant signal naturally present in the test room. These methods are referred to as blind methods in the literature and the maximum likelihood

\footnotetext{
a) Also at: School of Electrical and Computer Engineering, RMIT University, Melbourne, Australia. Electronic mail: wulifu@nuist.edu.cn
}

estimation (MLE) method is the predominant approach. ${ }^{5-7}$ In Ref. 9, a reverberation time estimation method based on single-channel blind room impulse response (RIR) identification is introduced, which can be treated as an approximation of the Schroeder's method. Most early literature related to these blind methods only address full band reverberation time estimation $^{5,6,9}$ and are, thus, not very useful for practical applications because sound absorption in rooms varies with frequency and the reverberation time is frequency dependent. ${ }^{7,8}$

One application of the blind methods in school classrooms and hospital wards estimated the reverberation time in 7 octave bands from $125 \mathrm{~Hz}$ to $8 \mathrm{kHz}^{7}$ In that study, the MLE method demonstrated similar accuracy to the standard measurement methods such as balloon bursts or swept-sine measurements, but it was found that the window length was a critical parameter in achieving accurate results. In another study, an all-pass warped filter-bank was employed to estimate frequency dependent reverberation time and this was shown to result in a more accurate reverberation time estimate at lower frequencies. ${ }^{8}$

The MLE method can be considered as an extension of the interrupted noise method and its precision is affected by factors such as the number of long pause gaps (segments where a speaker is silent), sharp offsets (the segments that the speech ceases abruptly), and statistical processing. ${ }^{5,6}$ In 
comparison with the interrupted noise method, Schroeder's method is preferred because it theoretically represents an average of an infinite number of interrupted noise measurements. Hence, if the RIR is indentified from the reverberant speech signal, the reverberation time can be estimated from that identified RIR using Schroeder's method. ${ }^{9}$

Taking the two aforementioned factors into consideration, the main objective of this paper is to evaluate reverberation time estimation performance in different octave bands on the basis of backward integration of the adaptively identified RIR. The RIR is identified under two different conditions, namely, (1) where the anechoic (also referred to as "clean" or "dry" in some literature) and reverberant signals are known a priori (i.e., the ideal condition), and the RIR can be identified using the well-established system identification scheme; (2) the anechoic signal is unknown and only the reverberant speech signal is available. In this case, blind identification of the RIR is used to establish a reverberation time estimate. ${ }^{9}$ In the remainder of this paper Sec. II describes the proposed approaches to reverberation time estimation, while details of the simulations and experiments are given in Sec. III. The results are summarised and the paper is concluded in Sec. IV.

\section{METHOD}

The reverberant signal $x(n)$ received in a room free from noise can be modeled by the convolution of the anechoic signal $s(n)$ with the RIR $\mathbf{h}(n)$,

$$
x(n)=\sum_{k=0}^{N-1} h(k) s(n-k),
$$

where $\mathbf{h}(n)$ is an $N$-tap finite impulse response (FIR) filter.

An ideal condition is considered first, where the anechoic and reverberant signals are known a priori, i.e., $x(n)$ and $s(n)$ are known in Eq. (1) and the RIR $\mathbf{h}(n)$ can be obtained using well-established system identification methods. There are many techniques for system identification, in such a case the normalized least mean square (NLMS) algorithm is used to identify the RIR due to its easy implementation and good performance. ${ }^{9}$ The RIR estimation, $\hat{\mathbf{h}}(n)$, is an $M$-tap FIR filter which is updated by the NLMS algorithm according to 9

$$
\hat{\mathbf{h}}(n+1)=\hat{\mathbf{h}}(n)+\mu_{1} \frac{\mathbf{s}(n)}{\mathbf{s}^{T}(n) \mathbf{s}(n)} e(n),
$$

where $\mu_{1}$ is the step size and $\mathbf{s}(n)=[s(n), s(n-1), \ldots, s(n$ $-M+1)]^{T}$. $e(n)$ is the difference between the observed reverberant signal $x(n)$ and the estimated reverberant signal $\hat{x}(n)$, i.e., $e(n)=x(n)-\hat{x}(n)$. The NLMS algorithm is a variant of the least mean square (LMS) algorithm and it solves the problem that the LMS algorithm is sensitive to the scaling of its input $[\mathbf{s}(n)]$ by normalizing with the power of the input. Table I lists the main steps for the NLMS algorithm and Fig. 1 demonstrates the immediate results of the RIR estimation $\hat{\mathbf{h}}(n)$, where the original RIR $\mathbf{h}(n)$ is measured from a lecture room. ${ }^{14}$ Figure 1(a) shows the anechoic
TABLE I. The main steps of the NLMS algorithm.

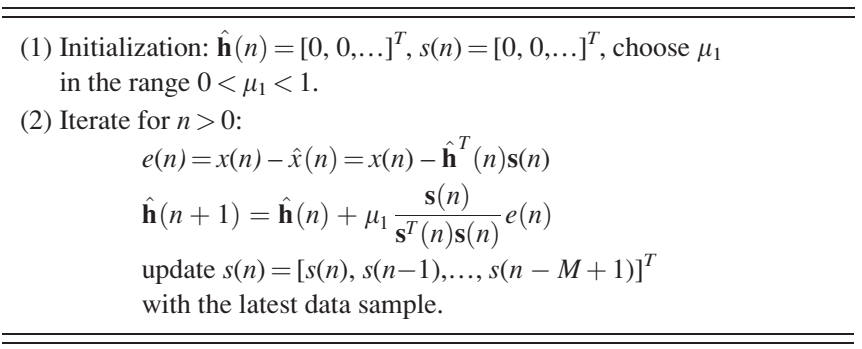

speech $[s(n)$ in Eq. (1)], Fig. 1(b) shows the reverberant speech $[x(n)$ in Eq. (1)], Fig. 1(c) shows the estimated reverberant speech $\hat{x}(n)$, and Fig. 1(d) shows the RIR estimation $\hat{\mathbf{h}}(n)$ using the NLMS algorithm.

After the RIR estimation $\hat{\mathbf{h}}(n)$ is obtained, the reverberation time in each octave band is determined by filtering $\hat{\mathbf{h}}(n)$ with a set of octave band-pass filters ${ }^{10}$ and applying Schroeder's method. ${ }^{3}$ Figure 2 shows the main procedure for reverberation time estimation under this ideal condition. The inputs are the anechoic signal $s(n)$ and the reverberant signal $x(n)$, and the output is the estimated reverberation times in each specific octave band. The sampling frequency of the anechoic signal used in this paper was $16 \mathrm{kHz}$, and the reverberation time was estimated in 7 octave bands with centre frequencies of $63,125,250,500,1000,2000$, and $4000 \mathrm{~Hz}$.

If only the reverberant speech signal can be observed and the anechoic signal is unknown, the anechoic signal must be estimated first. In such a case, $s(n)$ is estimated by dereverberating $x(n)$ on the basis of the maximization of the third-order moment (skewness) of the linear prediction (LP) residual. It is known that skewness is more effective as a statistical basis for dereverberation than the previously used kurtosis in terms of noise-related degradation. ${ }^{11}$ Figure 3 shows the diagram of the proposed algorithm for anechoic speech signal estimation.

Since reverberation affects the LP residual more significantly than the LP coefficients, and the LP filter can approximately remove the vocal tract contribution to the reverberant speech, the LP residual $r(n)$ is obtained first in Fig. 3 on the basis of LP analysis of the reverberant speech $x(n) .{ }^{11}$ In the figure, $\mathbf{a}(n)$ is the LP filter and $\mathbf{w}(n)$ is the dereverberation filter $\left(L\right.$-tap FIR) adaptively updated according to ${ }^{11}$

$$
\begin{aligned}
\mathbf{w}(n+1)= & \mathbf{w}(n) \\
& +\mu_{2} \frac{\hat{r}^{2}(n) E\left\{\hat{r}^{2}(n)\right\}-\hat{r}(n) E\left\{\hat{r}^{3}(n)\right\}}{E^{2.5}\left\{\hat{r}^{2}(n)\right\}} \mathbf{r}(n),
\end{aligned}
$$

where $\mu_{2}$ is the step size and vector $\mathbf{r}(\mathrm{n})=[r(n)$, $r(n-1), \ldots, r(n-L+1)]^{T}$. The expected values can then be calculated recursively using Eqs. (4) and (5):

$$
\begin{aligned}
& E\left\{\hat{r}^{2}(n)\right\}=\beta E\left\{\hat{r}^{2}(n-1)\right\}+(1-\beta) \hat{r}^{2}(n-1), \\
& E\left\{\hat{r}^{3}(n)\right\}=\beta E\left\{\hat{r}^{3}(n-1)\right\}+(1-\beta) \hat{r}^{3}(n-1),
\end{aligned}
$$

where $\beta$ is the weighing factor in the recursive update which controls the smoothness of the moment estimation. 


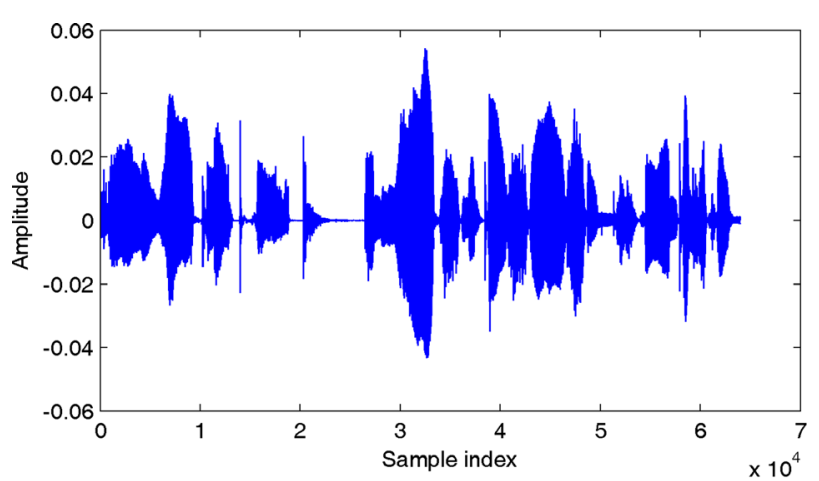

(a)

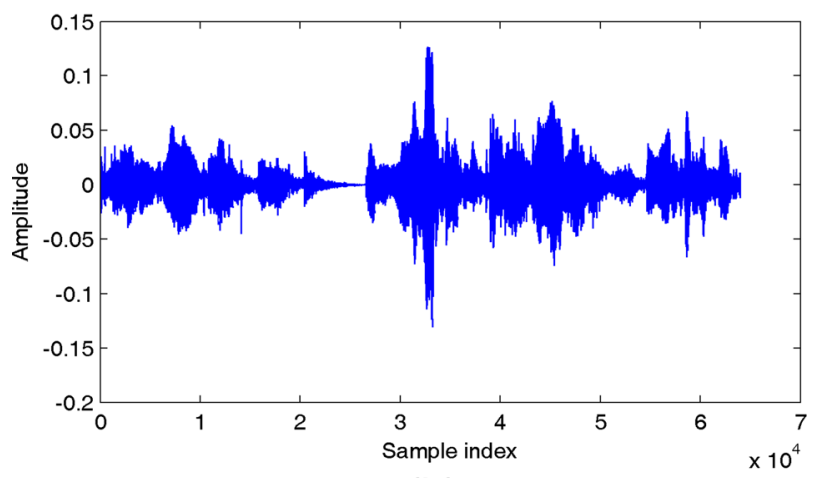

(b)

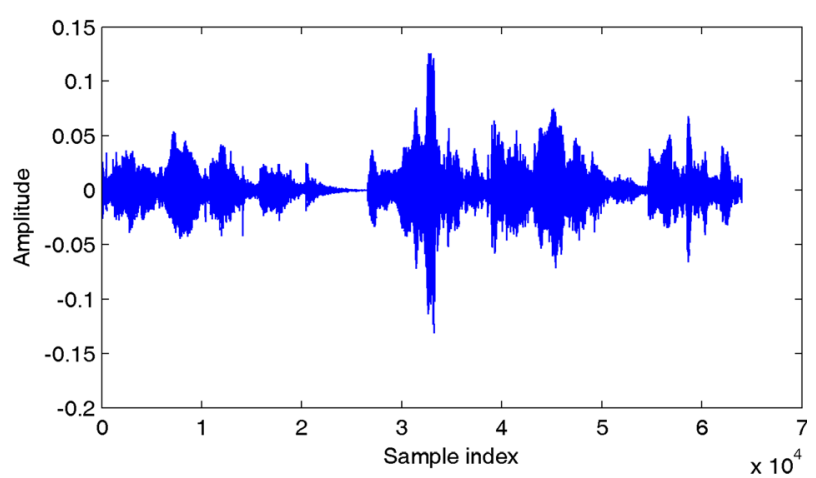

(c)

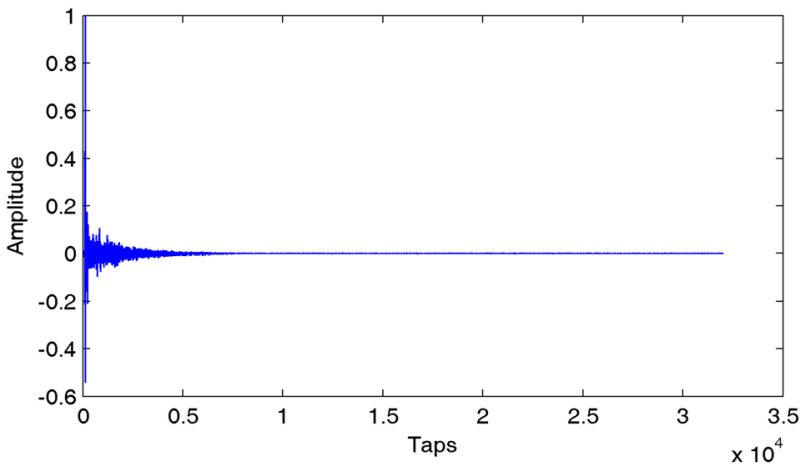

(d)

FIG. 1. (Color online) Illustration of RIR estimation under the ideal condition, (a) the anechoic speech, (b) the reverberant speech, (c) the estimated reverberant speech, (d) the estimated RIR.

The optimal parameters such as the weighing factor $\beta$, the step size $\mu_{1}$ and $\mu_{2}$ in each algorithm are difficult to select, and were chosen by trial and error in the paper to ensure that the algorithms were stable, converged rapidly, and provided satisfactory performance. The values used in the following evaluations were $\beta=0.9, \mu_{1}=0.1$, and $\mu_{2}=1 \times 10^{-5}$. The LP residual was calculated using a 20 tap LP filter where $\mathbf{a}(n)$ was computed with the Levinson-Durbin algorithm. The frame length and frame shift were 32 and $16 \mathrm{~ms}$, respectively, and a Hamming window was used as the window function.

Figure 4 illustrates the immediate results of the dereverberation by maximizing skewness of the LP residual, where the data are the same as the data in Fig. 1. Figure 4(a) shows the LP residual of the reverberant speech $[r(n)$ in Fig. 3], Fig. 4(b) shows the LP residual after maximizing the skewness $[\hat{r}(n)$ in Fig. 3], and Fig. 4(c) shows the estimated anechoic speech $\hat{s}(n)$.

After the anechoic speech signal $\hat{s}(n)$ was estimated and substituted for $s(n)$ in the ideal condition, the reverberation time in each octave band can be obtained. However, the presence of background noise is inevitable in real environments, under the "Idea" condition, the background noise degrades the performance of the NLMS algorithm, under the second condition, the background noise not only deteriorates the performance of the NLMS algorithm but also the performance of the dereverberation algorithm. It has been found that the background noise alters the positions of the stationary points of the skewness-based dereverberation approach since additive noise shifts the speech signals towards a Gaussian probability density function. ${ }^{11}$ Thus the estimation errors caused by the background noise are larger than that under the Idea condition. It is known that single-channel dereverberation technologies can hardly provide errorless anechoic speech signals, so there might be more bias in the estimation.

Since the background noise and single-channel dereverberation technologies both bring in the errors of RIR estimation, these estimation errors can be treated as noise in the measured RIR that distorts the reverberation time estimates. Several noise compensation methods have been proposed and a simplified version of the Lundeby method was employed in this paper and is described in Table II. ${ }^{12}$ The optimal $\theta$ in Table II is difficult to determine. A large value for $\theta$ excludes the noise distorted part in $\hat{\mathbf{h}}(n)$ and truncates $\hat{\mathbf{h}}(n)$ too much resulting in insufficient data for backward integration; on the contrary, a small value of $\theta$ provides more data for reverberation time estimation but with more noise, resulting in overestimated reverberation time.

Simulations show that $\theta=5-8$ is a good choice, and $\theta=6.5$ is used in the rest of the paper. Figure 5 illustrates the main steps of reverberation time estimation under the second condition. The length of the adaptive filter $\mathbf{w}(n)$ was chosen to be the same length as that of $\hat{\mathbf{h}}(n)$, which depends directly on the RIR length and relates to the reverberation time. It is not easy to select the correct tap length for different reverberation times and a small tap length is insufficient to cover the exponential decay sections of RIRs with high reverberation time. Thus, a large tap length (32000) was selected because this value gives coverage of reverberation time up to $2.0 \mathrm{~s}$. The truncation method described in Table II 


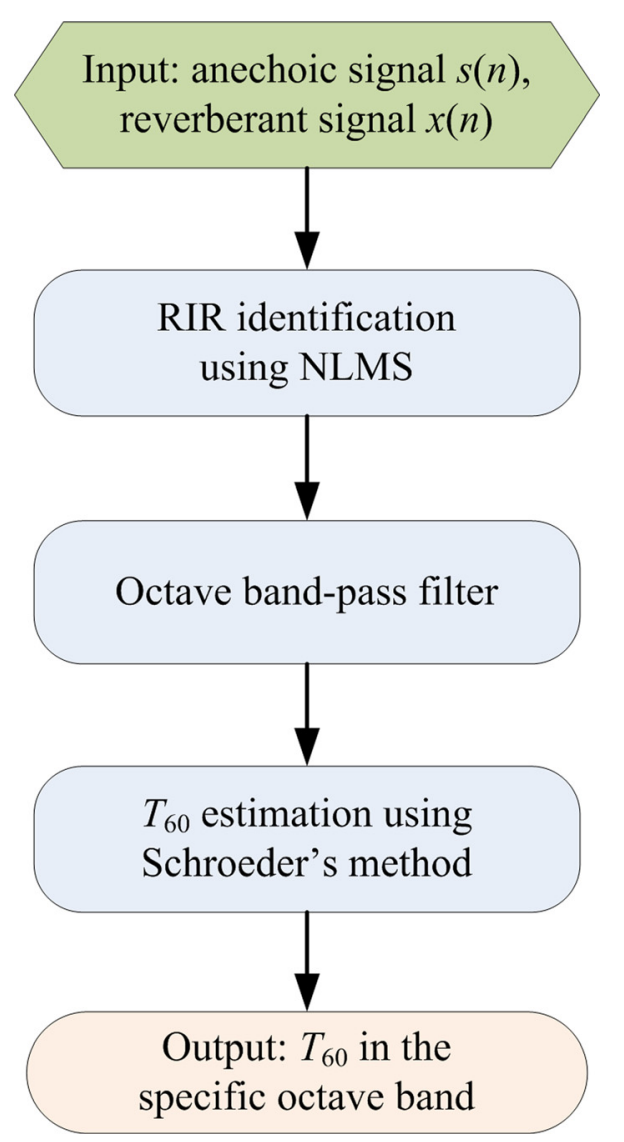

FIG. 2. (Color online) Summary of the procedure for reverberation time estimation under the ideal condition (both anechoic and reverberant signals are known a priori).

was used to choose the suitable RIR part for the reverberation time estimation.

Under the ideal condition, two kinds of anechoic signals, i.e., white noise and speech, were used for the evaluations. White noise was chosen as it is the commonly used signal in system identification and the results obtained can thus provide a comparative reference. Speech was selected from the TIMIT corpus which contains English sentences spoken by different male and female speakers and has been frequently used in the reverberation time estimation literature. ${ }^{13}$ As the duration of the original sentences from the TIMIT corpus are only several seconds, sentences from each speaker are concatenated to form utterances of $90 \mathrm{~s}$. For the

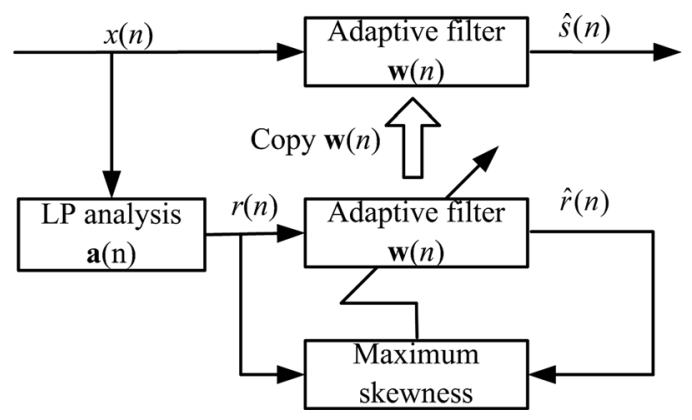

FIG. 3. Diagram of algorithm for anechoic speech estimation based on LP residual skewness maximization.

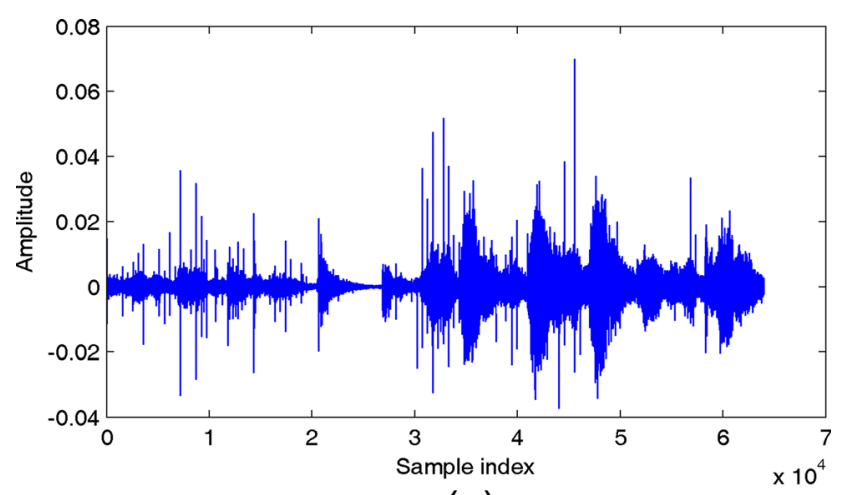

(a)

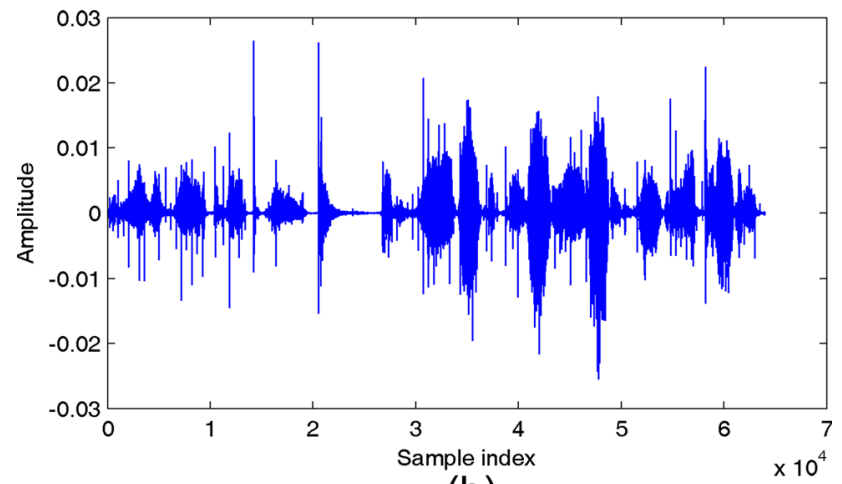

(b)

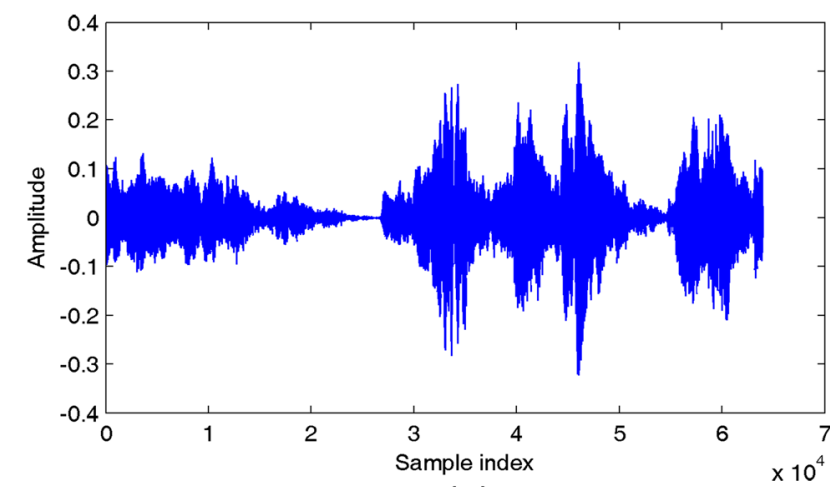

(c)

FIG. 4. (Color online) Illustration of anechoic speech estimation based on LP residual skewness maximization, (a) the LP residual of the reverberant speech, (b) the LP residual after maximizing the skewness, (c) the estimated anechoic speech.

second condition, only speech from the TIMIT corpus is used. The RIR dataset used in the simulations were real world measured RIRs from the Aachen Impulse Response database and included 5 spaces: lecture room, office room, meeting room, stairway, and Carolina Aula (a renovated monastery church). ${ }^{14}$ For the Aachen Impulse Response database, the positions of the loudspeaker and microphone are different, for example, the RIRs of the lecture room has been measured with typical auditorium furniture like desks and chairs, and the loudspeaker was placed at the lectern and the microphone was at different rows with increasing distance to the loudspeaker. Therefore the results shown in Sec. III have covered the effects of the positions of the loudspeaker and microphone, which is the average reverberation time of the room. 
TABLE II. A simplified Lundeby room impulse response truncation method for noise compensation.

(1) Calculate the squared amplitude of $\hat{\mathbf{h}}(n)$ and translate it to the logarithmic scale, i.e., $\chi(n)=10 \log _{10} \hat{\mathbf{h}}^{2}(n)$.

(2) Segment $\chi(n)$ into frames of length $30 \mathrm{~ms}$ and compute the average $[\bar{\chi}(k), k$ is the frame index] of each frame.

(3) Estimate the noise level of the last $10 \%$ frames because the amplitude of noise-free RIR tail is very small and the estimation errors dominate the tail of the impulse response $\hat{\mathbf{h}}(n)$.

(4) Find the frame index $k$ from tail to the head where $\bar{\chi}(k)$ exceeds that of the estimated noise level by a certain amount (defined $\theta \mathrm{dB}$ here).

(5) Utilize the truncated part from the first frame to the $k$ th frame of $\hat{\mathbf{h}}(n)$ as the input of the standard Schroeder method to estimate reverberation time.

\section{RESULTS AND DISCUSSIONS}

The estimation results are presented in Fig. 6, where in the legends, "Benchmark" represents the correct value calculated directly from the original RIRs of the Aachen Impulse Response database and "Speech" indicates the values under the ideal condition with the anechoic signal being speech, "White noise" indicates the values under the ideal condition with the anechoic signal being white noise and "Proposed" identifies values obtained under the second condition with only the reverberant speech being known. These graphs show the mean values along with the error bars calculated on the basis of 240 independent trials.

The absolute value of relative error (absolute relative error) $\varepsilon_{\mathrm{r}}$ between the "Benchmark" value and the estimated value is listed in Table III, and was calculated by

$$
\varepsilon_{\mathrm{r}}=\frac{\left|T_{60}-\bar{T}_{60}\right|}{T_{60}} \times 100 \%
$$

where " $T_{60}$ " is the value marked as "Benchmark" bar in Fig. 6 and " $\bar{T}_{60}$ " is the average value of the estimated $T_{60}$ marked as "Speech," "White noise," and "Proposed" bars in Fig. 6.

Under the ideal condition, white noise was used as the anechoic signal. For the lecture room and the stairway, the

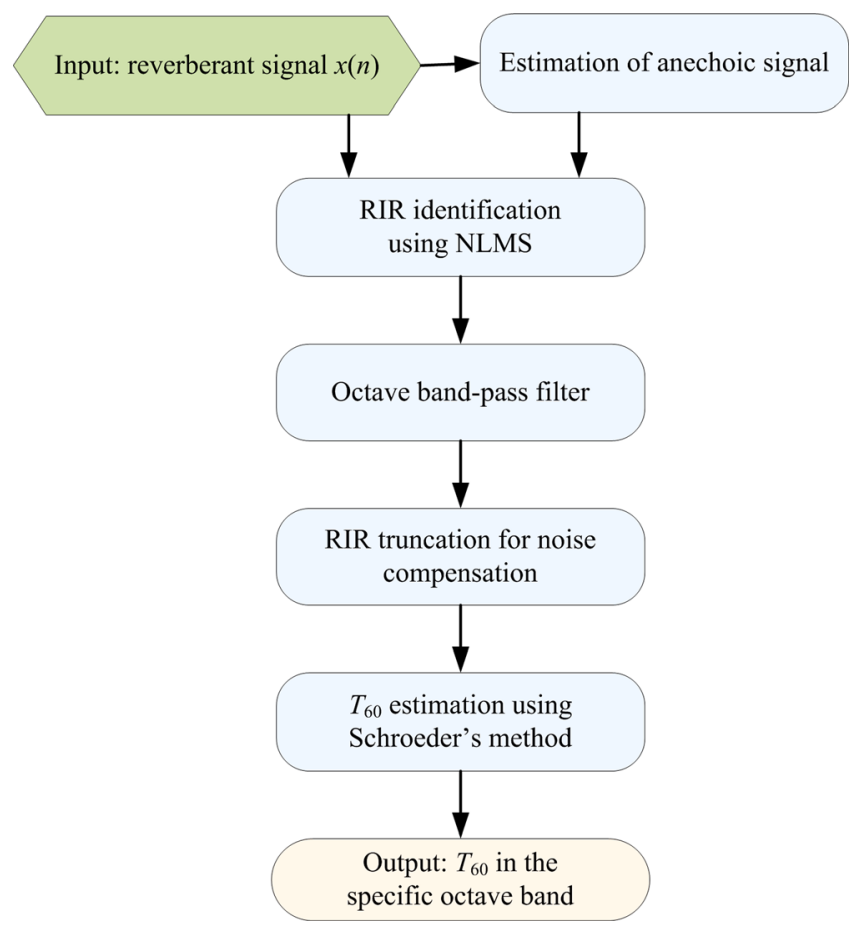

FIG. 5. (Color online) Approach to reverberation time estimation under the second condition (only the reverberant signal is known a priori). absolute relative errors are no greater than $0.3 \%$; for the office room, the absolute relative error at $63 \mathrm{~Hz}$ octave band is $1.1 \%$, the other absolute relative errors are all less than or equal to $0.3 \%$; for the meeting room, the absolute relative error at $63 \mathrm{~Hz}$ octave band is $2.7 \%$, the other absolute relative errors are all less than or equal to $0.4 \%$; for the Carolina Aula, the absolute relative error at $4000 \mathrm{~Hz}$ octave band is $0.8 \%$, the other absolute relative errors are within the range $1.3 \%-6.0 \%$. Therefore, the performance with the white noise as the anechoic signal is quite accurate and the absolute relative errors are mostly less than $6 \%$.

Under the ideal condition when speech is used as the anechoic signal, the performance deteriorates. For the lecture room, the absolute relative error in the 63, 250, 500, 1000, and $4000 \mathrm{~Hz}$ octave bands are less than $1 \%$, but the absolute relative error at $125 \mathrm{~Hz}$ octave band is $19.4 \%$; for the office room, the absolute relative error for the 63, 250, 500, 1000, and $4000 \mathrm{~Hz}$ octave bands are less than $1.5 \%$, the absolute relative error at 125 and $2000 \mathrm{~Hz}$ octave bands are $5.0 \%$, and $7.3 \%$, respectively; for the meeting room, the absolute relative error in the $63,250,500$, and $1000 \mathrm{~Hz}$ octave bands are less than $2.5 \%$, the absolute relative error at 125 and $2000 \mathrm{~Hz}$ octave bands are $20.5 \%$ and $17.9 \%$, respectively; for the stairway, the absolute relative errors for the 125,250 , 500,1000 , and $2000 \mathrm{~Hz}$ octave bands are less than $9 \%$, the absolute relative error at 63 and $4000 \mathrm{~Hz}$ octave bands are $51.4 \%$ and $12.8 \%$, respectively; for the Carolina Aula, the absolute relative error from 125 to $4000 \mathrm{~Hz}$ octave band is within the range $1.3 \%-7.5 \%$, while the absolute relative error at $63 \mathrm{~Hz}$ octave band is $16.1 \%$. The performance that uses speech as the anechoic signal is somehow acceptable with most absolute relative errors less than $10 \%$.

The error bars in Fig. 6 show that the variance of the estimated reverberation time using speech is greater than that using white noise under the ideal condition because the statistical characteristics of white noise are stationary while the speech signal is non-stationary and the randomly selected sentences from TIMIT database lead to larger variance in the estimation. The results from Fig. 6 and Table III confirm that if the anechoic and reverberant speech signals are known $a$ priori, reverberation time estimation based on the RIR identification is feasible and reliable.

Under the second condition where only reverberant speech is used, the maximum absolute relative errors of the five different rooms all occurred at the $63 \mathrm{~Hz}$ octave band and the absolute relative errors are more than $80 \%$. For the lecture room, the absolute relative errors in the 125,250 , 1000,2000 , and $4000 \mathrm{~Hz}$ octave bands are all less than $25 \%$; for the office room, the absolute relative errors excepting that of the $63 \mathrm{~Hz}$ octave band are all less than $25 \%$ and the 


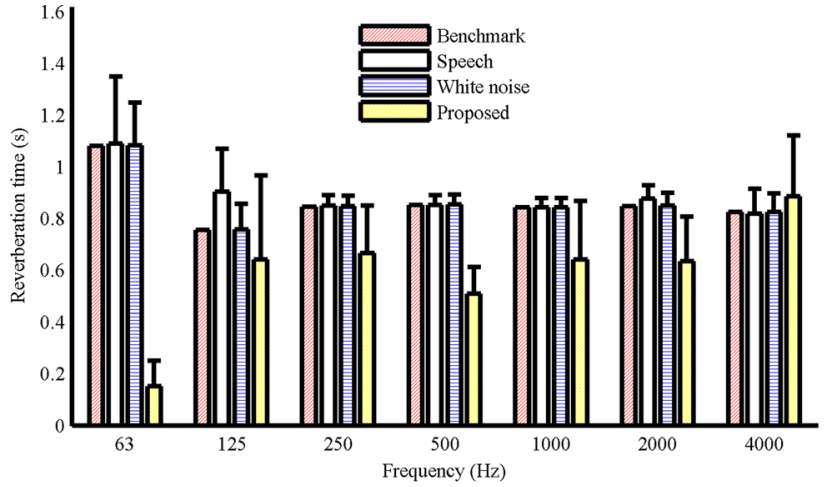

(a)

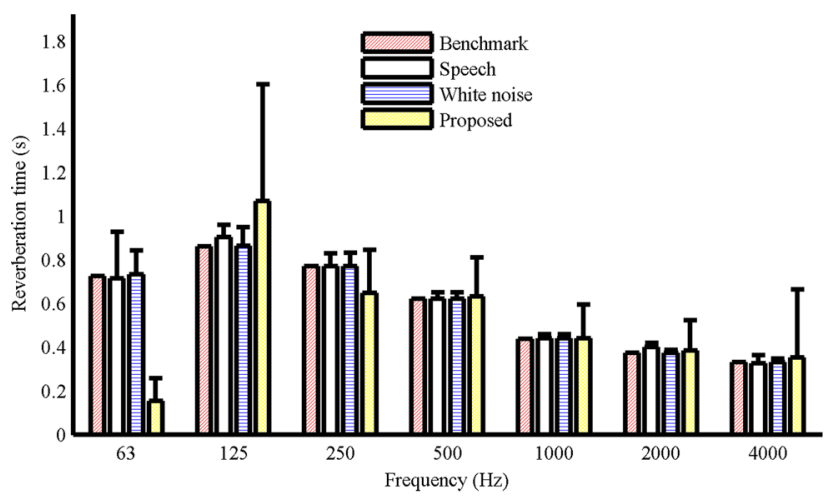

(b)

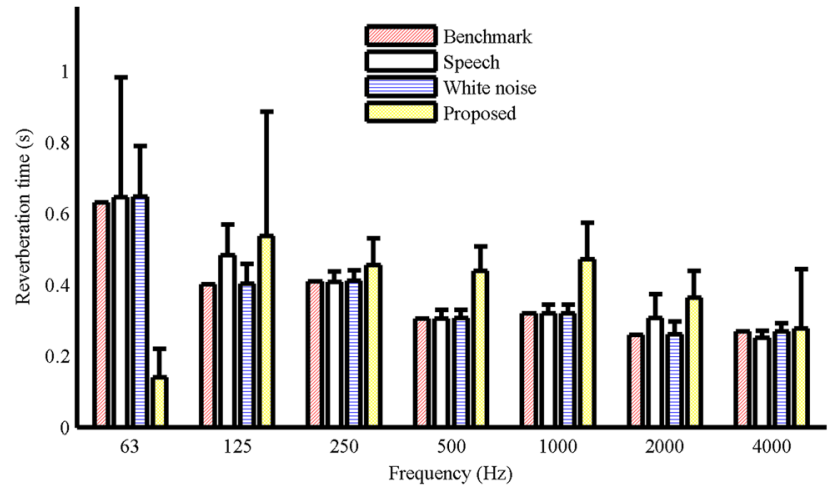

(c)

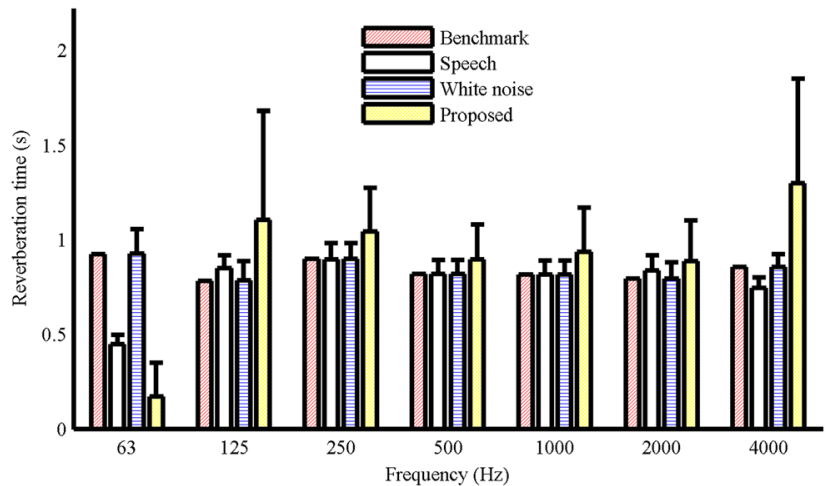

(d)

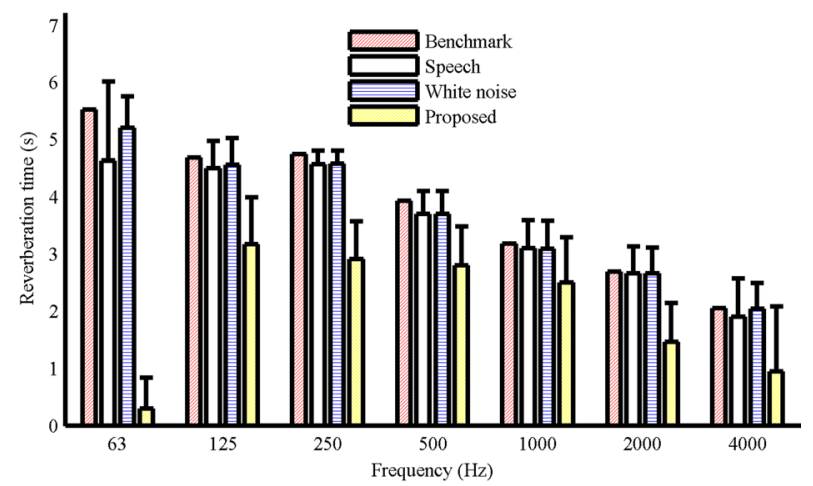

(e)

FIG. 6. (Color online) Reverberation time estimation for 5 different spaces: (a) lecture room, (b) office room, (c) meeting room, (d) stairway, and (e) Carolina Aula.

absolute relative errors in the 500,1000, and $2000 \mathrm{~Hz}$ octave bands are even less than 3.0\%; for the meeting room, the absolute relative errors in the 250 and $4000 \mathrm{~Hz}$ octave bands are less than $12 \%$ and the others are within the range $30 \%-48 \%$; for the stairway, the absolute relative errors for the 250, 5001000 , and $2000 \mathrm{~Hz}$ octave bands are less than $15 \%$; for the Carolina Aula, the absolute relative errors in the $125,250,500$, and $1000 \mathrm{~Hz}$ octave bands are within the range $20 \%-40 \%$. In comparison with the error bars in Fig. 6 , the estimation variance under the second condition is much larger than that under the ideal condition, therefore, a larger number of estimations should be carried out and averaged to obtain a reliable reverberation time in practical measurements.

The average of the absolute relative errors over the five spaces in 7 octave bands is calculated and plotted in Fig. 7.
The average of the absolute relative errors under the ideal condition with white noise are all less than $2 \%$, but become less than $15 \%$ when the anechoic signal is speech. The largest error occurs in the low frequency bands (such as the 63 and $125 \mathrm{~Hz}$ bands) and the high frequency band $(4000 \mathrm{~Hz})$. The reason for this is that the energy of speech signals is predominantly in the range $300-3400 \mathrm{~Hz}$ so there is insufficient signal for identifying the RIR accurately in the frequency bands outside that range. Under the second condition, the average of the absolute relative errors is generally larger than $20 \%$. This is because there is insufficient information from the single-channel RIR identification, hence the precision of the estimated RIR is limited and the relative error under the second condition is larger than that achieved under the ideal condition.

To further verify the performance under the second condition, the reverberation time of two different meeting rooms 
TABLE III. Absolute relative error $(\%)$ for speech, white noise, and the proposed cases (reverberant speech only).

\begin{tabular}{lccccccccc}
\hline \hline Octave band centre frequency (Hz) & 63 & 125 & 250 & 500 & 1000 & 2000 & 4000 \\
\hline Lecture room & Speech & 0.9 & 19.4 & 0.4 & 0.0 & 0.2 & 3.5 & 0.7 \\
& White noise & 0.3 & 0.3 & 0.2 & 0.2 & 0.2 & 0.2 & 0.2 \\
& Proposed & 85.9 & 15.2 & 21.4 & 40.2 & 23.8 & 25.0 & 7.3 \\
Office room & Speech & 1.5 & 5.0 & 0.0 & 0.0 & 0.1 & 7.3 & 1.2 \\
& White noise & 1.1 & 0.3 & 0.3 & 0.1 & 0.1 & 0.1 & 0.3 \\
& Proposed & 78.7 & 24.4 & 15.7 & 2.1 & 0.5 & 3.0 & 7.0 \\
Meeting room & Speech & 2.3 & 20.5 & 0.3 & 0.0 & 0.1 & 17.9 & 6.8 \\
& White noise & 2.7 & 0.4 & 0.2 & 0.2 & 0.2 & 0.3 & 0.3 \\
& Proposed & 77.7 & 33.6 & 11.3 & 43.8 & 47.5 & 40.0 & 2.9 \\
Stairway & Speech & 51.4 & 8.9 & 0.1 & 0.0 & 0.0 & 5.4 & 12.8 \\
& White noise & 0.2 & 0.3 & 0.1 & 0.1 & 0.1 & 0.1 & 0.1 \\
& Proposed & 81.7 & 41.4 & 16.1 & 9.6 & 14.8 & 11.9 & 51.8 \\
Carolina Aula & Speech & 16.1 & 3.8 & 3.6 & 6.0 & 2.2 & 1.3 & 7.5 \\
& White noise & 5.9 & 2.5 & 3.5 & 6.0 & 2.6 & 1.3 & 0.8 \\
& Proposed & 94.6 & 32.4 & 38.8 & 28.8 & 21.5 & 45.9 & 53.8 \\
\hline
\end{tabular}

in RMIT University were measured. The first meeting room was a normal "shoe-box" shape with $4.7 \mathrm{~m}$ (long) $\times 2.9 \mathrm{~m}$ (wide) $\times 2.7 \mathrm{~m}$ (high), and the second measured room, named "Fabpod," is a non-regular meeting pod with an open ceiling. ${ }^{15}$ The walls of the approximately $4 \mathrm{~m} \times 4 \mathrm{~m} \times 3 \mathrm{~m}$ Fabpod are curved and made of a number of hyperboloid cells. The experimental settings are shown in Fig. 8 and the height of both the sound source and the microphone for the measurements are $1.2 \mathrm{~m}$. The system is first calibrated at $94 \mathrm{~dB}$ at $1000 \mathrm{~Hz}$ with a B\&K type 4231 calibrator. The impulse response was measured using the exponential sweep frequency approach and the B\&K Dirac 6.0 software. The signal was generated by that software package, and reproduced through a power amplifier (Nor 280) and omnidirectional sound source (Norsonic Nor 276). The reverberant signal was captured using a B\&K type 4189 microphone and analysed by the Dirac software. The measurements were repeated 30 times and the impulse responses were recorded with reverberation times then computed from the impulse responses in the individual octave bands from 63 to $4000 \mathrm{~Hz}$.

The reverberation time obtained from the measurements and the proposed method is shown in Fig. 9. From the error bars, it can be seen that the fluctuation of measurement values

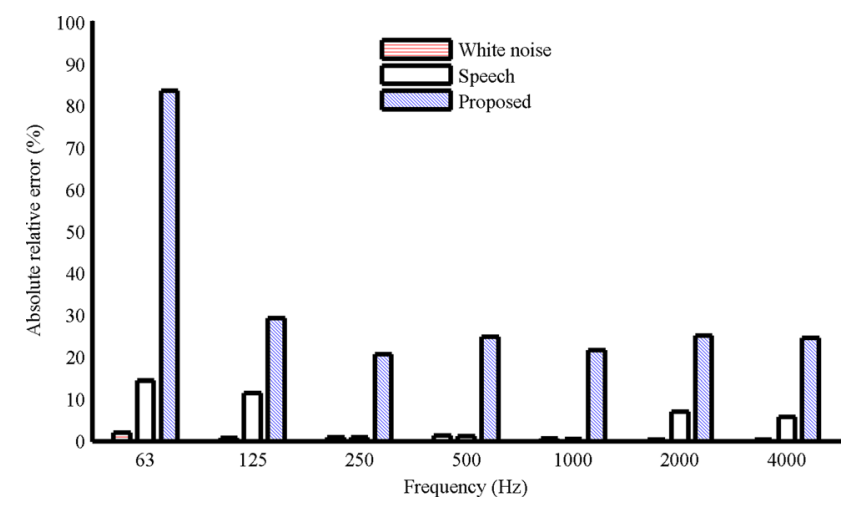

FIG. 7. (Color online) Frequency dependent relative error under the two conditions (averaged over five spaces).

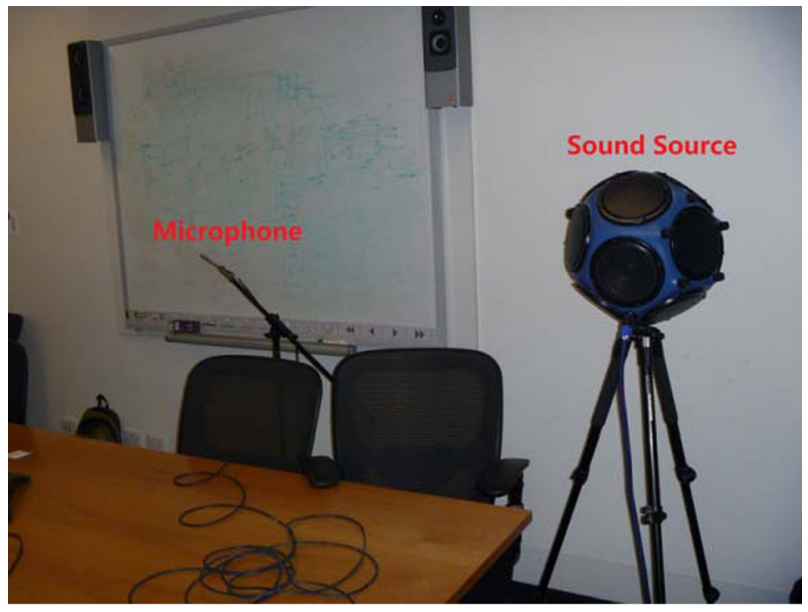

(a)

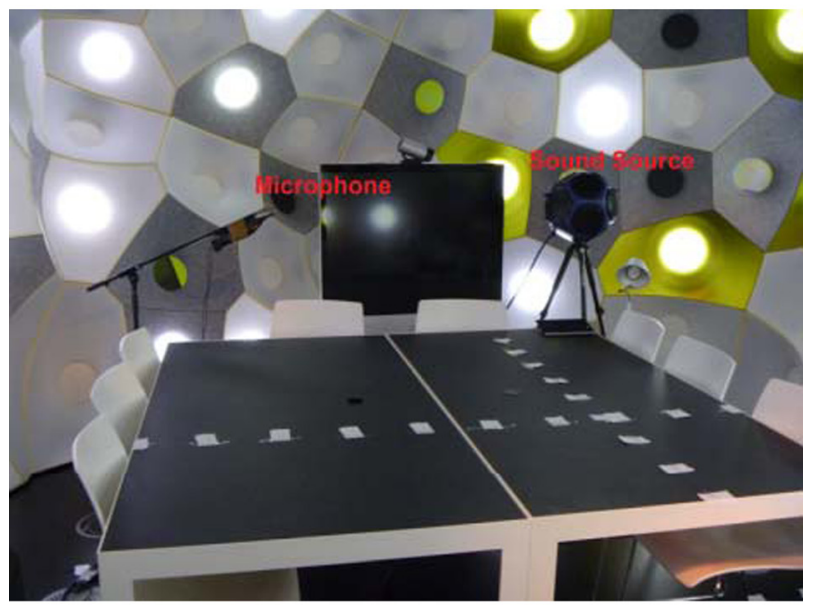

(b)

FIG. 8. (Color online) Reverberation time measurement setup in (a) "shoebox" shape meeting room and (b) Fabpod.

in the $63 \mathrm{~Hz}$ octave band is larger than that of the other 6 octave bands. The error between the measurement and proposed method for the $63 \mathrm{~Hz}$ octave band is also larger than that in the remaining bands; as mentioned previously, this is predominantly caused by the main energy of speech signal being concentrated above $300 \mathrm{~Hz}$ and it is thus difficult to estimate the RIR component reliably in the $63 \mathrm{~Hz}$ octave band. In comparison with the measurements, the error bars show that the estimation fluctuation of the proposed method is larger than that of the measurements. The absolute relative errors of the 7 octave bands in the shoe-box shape meeting room are $61.5 \%, 6.3 \%, 19.2 \%, 19.4 \%, 8.5 \%, 8.6 \%$, and $13.4 \%$, respectively, while those in the Fabpod are $64.3 \%, 5.0 \%, 4.2 \%$, $8.5 \%, 21.9 \%, 4.6 \%$, and $11.1 \%$, respectively. Because there is no explicit statement for the acceptable error margin when estimating $T_{60}$ in the standards such as ISO 3382, it is hard to claim whether the estimation error is too large or not. ${ }^{3}$ The results show that for the environments with medium-range reverberation times such as the office room in Figs. 6(b), 6(d), and $9(\mathrm{a})$, the proposed method is able to provide reasonable estimation for the $250,500,1000$, and $2000 \mathrm{~Hz}$ octave bands with an absolute relative error less than $20 \%$. 


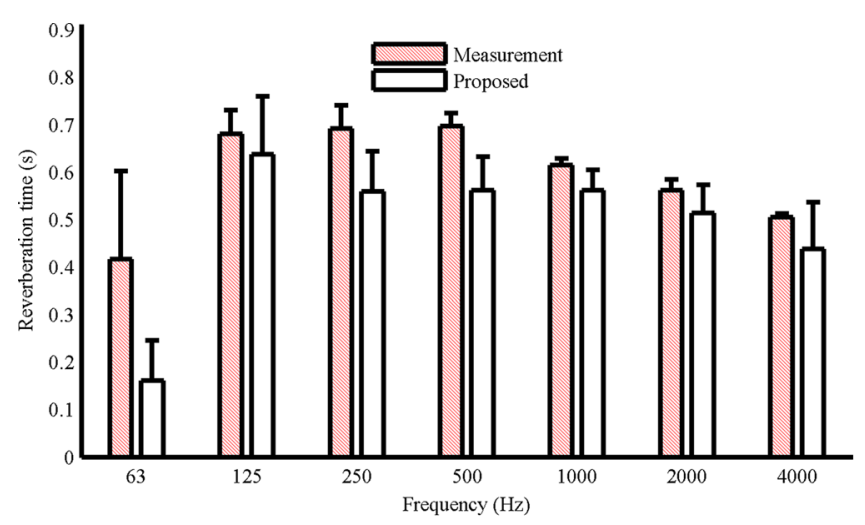

(a)

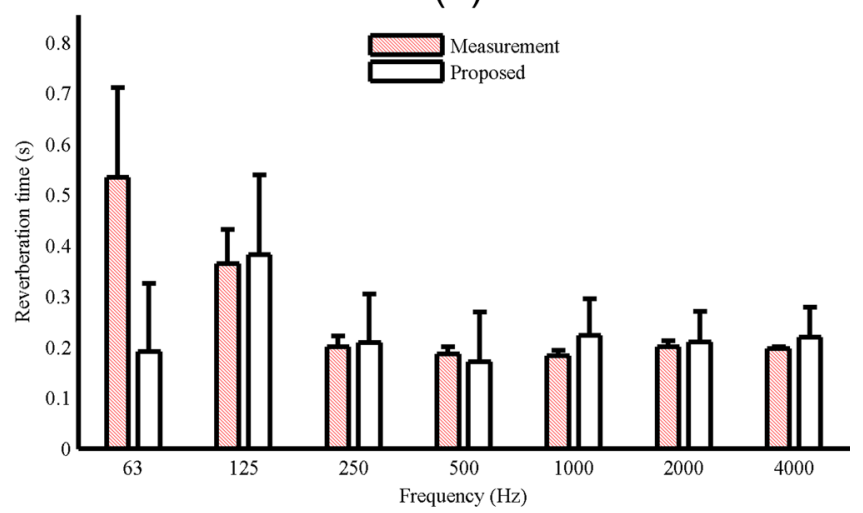

(b)

FIG. 9. (Color online) Performance comparison between the real reverberation time measurements and the proposed method in (a) "shoe-box" shape meeting room and (b) Fabpod.

\section{CONCLUSIONS}

Under the ideal condition where both the anechoic and reverberant signals are known a priori, a system identification scheme can be used to identify the RIR and then reverberation time can be estimated. If only the reverberant speech signal is observed and available, an approach to identify RIR blindly based on the skewness of LP residual maximization has been presented for reverberation time estimation. Simulations show that under the ideal condition, using speech and white noise as the anechoic signal the technique can estimate the reverberation time reliably, and the average relative error in 7 octave bands are mainly less than $15 \%$. Under the second condition, where only the reverberant speech signal is available, the estimation accuracy is worse than that under the ideal condition. The average relative error in the $63 \mathrm{~Hz}$ octave band is greater than $80 \%$, the other relative errors are mainly within the interval $20 \%-30 \%$, and the fluctuation of the estimated reverberation time from the proposed method is larger than those achieved under the ideal condition. The relative error in low frequency octave bands is usually larger than that in high octave bands. Reverberation time measurements in real rooms verified these findings.

There are still some challenges that require further work. For example, define an effective measure to evaluate the mismatch between the estimated room impulse responses and the idea ones, this measure will help to remove the big outliers of the reverberation time estimation; Improve the blind reverberation time estimation performance with background noise, if the background noise is seen as the additive, then some speech enhancement methods may be used to remove the background noise to a certain extent, i.e., a noise cancelling can be carried out before the dereverberation.

\section{ACKNOWLEDGMENTS}

This project was funded by National Science Foundation of China $(11504176,11474163)$ and the Priority Academic Program Development of Jiangsu Higher Education Institutions. This research was also supported under Australian Research Council's Linkage Projects funding scheme (LP140100987). Thanks for Sipei Zhao's help on the measurements in RMIT University.

${ }^{1}$ H. Kuttruff, Room Acoustics, 5th ed. (Taylor \& Francis, New York, 2009), Chap. 3, pp. 67-100.

${ }^{2}$ N. R. Shabtai, B. Rafaely, and Y. Zigel, "The effect of reverberation on the performance of cepstral mean subtraction in speaker verification," Appl. Acoust. 72, 124-126 (2011).

${ }^{3}$ ISO 3382:1997, Acoustics-Measurement of the reverberation time of rooms with reference to other acoustical parameters, 2nd ed. (International Organization for Standardization, Geneva, Switzerland, 1997).

${ }^{4}$ R. Dragonetti, C. Ianniello, and R. A. Romano, "Reverberation time measurement by the product of two room impulse responses," Appl. Acoust. 70, 231-243 (2009).

${ }^{5}$ R. Ratnam, D. L. Jones, B. C. Wheeler, W. D. O’Brien, Jr., C. R. Lansing, and A. S. Feng, "Blind estimation of reverberation time," J. Acoust. Soc. Am. 114(5), 2877-2892 (2003).

${ }^{6}$ H. W. Löllmann, E. Yilmaz, M. Jeub, and P. Vary, "An improved algorithm for blind reverberation time estimation," in Proceedings of Int. Workshop Acoust. Echo Noise Control, Tel Aviv, Israel (2010).

${ }^{7}$ P. Kendrick, N. Shiers, R. Conetta, T. J. Cox, B. M. Shield, and C. Mydlarz, "Blind estimation of reverberation time in classrooms and hospital wards," Appl. Acoust. 73, 770-780 (2012).

${ }^{8} \mathrm{H}$. W. Löllmann and P. Vary, "Estimation of the frequency dependent reverberation time by means of warped filter-banks," in Proceedings of the IEEE International Conference on Acoustics, Speech, and Signal Processing, Prague, Czech Republic (2011).

${ }^{9}$ L. Wu, X. Qiu, I. Burnett, and Y. Guo, "Reverberation time estimation from speech signals based on blind room impulse response identification," J. Acoust. Soc. Am. 138(2), 731-734 (2015).

${ }^{10}$ ANSI S1.11-1986, Specification for Octave-Band and Fraction-OctaveBand Analog and Digital Filters (American National Standards Institute, Washington, DC, 1986).

${ }^{11}$ S. Mosayyebpour, H. Sheikhzadeh, T. A. Gulliver, and M. Esmaeili, "Single-microphone LP residual skewness-based inverse filtering of the room impulse response," IEEE Trans. Audio Speech Lang. Process. 20(5), 1617-1632 (2012).

${ }^{12}$ A. Lundeby, T. E. Vigran, H. Bietz, and M. Vorlander, "Uncertainties of measurements in room acoustics," Acustica 81(4), 344-355 (1995).

${ }^{13}$ J. S. Garofolo, Getting Started with the DARPA TIMIT CD-ROM: An Acoustic-Phonetic Continuous Speech Database (National Institute of Standards and Technology, Gaithersburg, MD, 1993).

${ }^{14}$ M. Jeub, M. Schafer, and P. Vary, "A binaural impulse response database for evaluation of dereverberation algorithms," in Proceedings of Intl. Conf. Digital Signal Process., Santorini, Greece (2009).

${ }^{15}$ S. Zhao, X. Qiu, E. Cheng, I. Burnett, N. Williams, J. Burry, and M. Burry, "Sound quality inside small meeting rooms with different room shape and fine structures," Appl. Acoust. 93, 65-74 (2015). 